\title{
Comparative Analysis of
} WUSCHEL-Related Homeobox Genes Revealed Their Parent-of-Origin and Cell Type-Specific Expression Pattern During Early Embryogenesis in Tobacco

OPEN ACCESS

Edited by:

Liwen Jiang,

The Chinese University of Hong Kong,

Hong Kong

Reviewed by:

Chengwei Yang

South China Normal University, China

Xiaohong Zhuang,

The Chinese University of Hong Kong,

Hong Kong

*Correspondence:

Peng Zhao

pzhao2000@whu.edu.cn

Specialty section:

This article was submitted to

Plant Cell Biology,

a section of the journal

Frontiers in Plant Science

Received: 05 December 2017

Accepted: 22 February 2018

Published: 08 March 2018

Citation:

Zhou X, Guo Y, Zhao P and Sun $M$

(2018) Comparative Analysis of WUSCHEL-Related Homeobox Genes

Revealed Their Parent-of-Origin and Cell Type-Specific Expression Pattern During Early Embryogenesis in

Tobacco. Front. Plant Sci. 9:311 doi: 10.3389/fp/s.2018.00311

\author{
Xuemei Zhou, Yingying Guo, Peng Zhao* and Meng-xiang Sun \\ State Key Laboratory of Hybrid Rice, College of Life Sciences, Wuhan University, Wuhan, China
}

WUSCHEL-related homeobox (WOX) gene is a plant-specific clade of homeobox transcription factors. Increasing evidences reveal that WOXs play critical roles in early embryogenesis, which involves zygote development, initiation of zygote division, and apical or basal cell lineage establishment. However, how WOXs regulate these developmental events remains largely unknown, and even detailed expression pattern in gametes and early proembryos is not yet available. Here, 13 WOX family genes were identified in Nicotiana tabacum genome. Comparative analysis of 13 WOX family genes with their homologs in Arabidopsis thaliana reveals relatively conserved expression pattern of WUS and WOX5 in shoot/root apical meristem. Whereas variations were also found, e.g., lacking homolog of WOX8 (a marker for suspensor cell) in tobacco genome and the expression of WOX2/WOX9 in both apical cell and basal cell. Transient transcriptional activity analysis revealed that WOXs in WUS clade have repressive activities for their target's transcription, whereas WOXs in ancient and intermediate clade have activation activities, giving a molecular basis for the phylogenetic classification of tobacco WOXs into three major clades. Expression pattern analysis revealed that some WOXs (e.g., WOX 13a) expressed in both male and female gametes and some WOXs (e.g., WOX 11 and WOX 13b) displayed the characteristics of parent-of-origin genes. Interestingly, some WOXs (e.g., WOX2 and WOX9), which are essential for early embryo patterning, were de novo transcribed in zygote, indicating relevant mechanism for embryo pattern formation is only established in zygote right after fertilization and not carried in by gametes. We also found that most WOXs displayed a stage-specific and cell type-specific expression pattern. Taken together, this work provides a detailed landscape of WOXs in tobacco during fertilization and early embryogenesis, which will facilitate the understanding of their specific roles in these critical developmental processes of embryogenesis.

Keywords: WUSCHEL-related homeobox gene, early embryo, apical cell, basal cell, transcription repressive activity, tobacco 


\section{INTRODUCTION}

Homeobox (HB) protein is a larger superfamily of eukaryotic transcription factors, which was first discovered in Drosophila melanogaster, and subsequently in many other eukaryotic organisms, ranging from sponges to vertebrates and mammals (Gehring et al., 1994). Homeobox proteins share a common homeodomain (HD) composed of 60 amino acids, which could recognize their binding sequences and regulate the expression of targeted genes in a precise spatial and temporal pattern, and exert various roles in different developmental processes, such as controlling of axial morphology and specifying segmental identity (Gehring et al., 1994; Pearson et al., 2005). Different types of $\mathrm{HB}$ genes have also been identified in plants, which exhibit diverse roles in plant developmental processes (Vollbrecht et al., 1991; Jain et al., 2008; Mukherjee et al., 2009). Among them, WUSCHEL-related homeobox (WOX) genes are distinguished as a plant-specific clade of $\mathrm{HB}$ transcription factors by phylogenetic relatedness of its $\mathrm{HD}$ from other $\mathrm{HB}$ transcription factors (van der Graaff et al., 2009).

WOX family genes were found to be ubiquitous present in the genomes of different plants, ranging from green algae to angiosperms (van der Graaff et al., 2009). However, the number of WOXs vary in plant genomes. WOX members expanded as the evolution of plants. There is only one WOX in the genome of green algae, whereas over 10 WOXs have been identified in the genome of angiosperms (Mukherjee et al., 2009). Phylogenetic analysis of WOX family genes from different species revealed that WOXs could be divided into three major clades including WUS clade, intermediate clade and ancient clade. Phylogenetic differences of WOX family genes between monocots and eudicots or between angiosperms and gymnosperms have also been revealed, respectively (van der Graaff et al., 2009).

Researches about WOX family genes in Arabidopsis thaliana revealed multiple roles of WOX family genes in different developmental processes, such as embryo patterning (Breuninger et al., 2008), stem-cell maintenance (Schoof et al., 2000; Leibfried et al., 2005; Forzani et al., 2014), floral transition (Lenhard et al., 2001), and so on. Fifteen WOX family genes have been identified in A. thaliana. Expression pattern analysis of them revealed that different members of WOX family display distinct expression pattern and comprise earliest cell fate markers in early embryogenesis (Haecker et al., 2004). WOX2 and WOX8 were found to be co-expressed in the zygote and confined to the apical cell and basal cell after zygote division, respectively. WOX9 was firstly detected in the basal cell and subsequently shifted into the descendants of the apical cell (Haecker et al., 2004). WOX2 was approved to act redundantly with WOX1, WOX3, and WOX5 in embryo patterning. WOX 8 and WOX9 were essential for development of apical and basal lineages of proembryo (Breuninger et al., 2008). After the establishment of the apical-basal pattern, another two WOX family genes, WUS and WOX5, could be detected in the shoot apical meristem (SAM) and root apical meristem (RAM), respectively (Schoof et al., 2000; Leibfried et al., 2005; Forzani et al., 2014).

Comparative analysis of WOX family genes between monocots and eudicots revealed that the expression pattern of several WOX family genes in SAM and RAM was partly similar, and the expression of these monocot orthologous genes appeared significantly delayed. Significant expression of $\mathrm{ZmWOX} 2 \mathrm{~A}$ and $Z m W O X 9 A / B$ could only be detected in embryos at early transition stage. The expression of WUS and WOX5 (ZmWUS1 and $Z m W O X 5)$ in Zea mays were detected as early as embryo already consists of more than 100 cells (Zhao et al., 2017). Some other variations in WOX family genes between monocots and eudicots have also been found. The most striking divergence is that no WOX8 has been identified in monocots to date. No similarities on the expression pattern of WOX4 from Z. mays and $A$. thaliana were detected (Nardmann et al., 2007). All these results implied that the expression of WOX family genes has undergone modifications during plant evolution.

Given important roles of WOX family genes in early embryogenesis, the detailed expression pattern of WOXs is a prerequisite for elucidating their specific roles in embryo initiation and cell fate determination during early embryogenesis. Although considerable works have been done in A. thaliana, the detailed expression pattern of all members of $W O X$ family in gametes, zygotes, and apical/basal cells is not yet investigated in any species. This expression pattern is necessary for evaluating the usability of these cell type-specific markers and elucidating the mechanism of embryo initiation and embryonic cell fate determination. Here, WOX family genes were firstly identified in Nicotiana tabacum, a model plant in eudicot clade, which displays a highly stereotyped, comparable and tractable cell division pattern during embryogenesis. Comparative analyses between $N$. tabacum and A. thaliana were carried out to gain insight into their conservation and differences. Especially, their specific expression in gametes and early proembryos was carefully investigated, providing a dynamic landscape of WOXs expression during fertilization and early embryogenesis.

\section{MATERIALS AND METHODS}

\section{Plant Materials}

$N$. tabacum were grown in a $25^{\circ} \mathrm{C}$ greenhouse under $16 / 8 \mathrm{~h}$ light/dark cycle. Nicotiana benthamiana were grown in a $21^{\circ} \mathrm{C}$ greenhouse under 14/10 h light/dark cycle.

\section{Identification of WUS Homeobox-Containing (WOX) Proteins}

To identify WOX family genes in N. tabacum, the program tBlastn using WOX family protein sequences from $A$. thaliana were performed in the draft genomes, mRNA, and EST sequences of N. tabacum, respectively. DNA fragments and mRNA sequences related to WOX family genes were collected and assembled using the ContigExpress program with a minimum overlap of 100 bases and above 90\% sequence identity in the overlap region. After assembly, redundant contigs related to WOX family genes were removed manually, and open reading frame (ORF) analysis of each contig was performed using OMEGA. Contigs with intact ORF were selected for further BLASTP analysis in National Center for Biotechnology Information. Contigs with HD domain were as WOX family candidates. Then, full-length cDNA of each candidate was 
obtained through reverse transcription-PCR (RT-PCR) with gene-specific primers at $3^{\prime}$ end and $5^{\prime}$ end, respectively.

\section{Protein Sequence and Phylogenetic Analysis}

To detect conserved domain of WOX family proteins, a multiple sequence alignment of protein sequences was conducted using the Clustal W program using the default multiple alignment parameters. Conserved motifs in WOX were analyzed on http://weblogo.berkeley.edu/logo.cgi. Signal peptide and nuclear localization signal predication were executed on SignalP server (Petersen et al., 2011) and cNLS Prediction, respectively. Threedimensional structure of each WOX was predicated on SWISSMODEL workspace (Arnold et al., 2006; Kiefer et al., 2009).

To construct a phylogenetic tree of WOXs from different plants, a genome-wide survey of WOX family genes in Glycine max, Oryza sativa, Z. mays, and Sorghum bicolor genomes was firstly executed, and sequences related to WOX family genes were collected. Then, a multiple sequence alignment of 84 WOX protein sequences from six different species was conducted using the Clustal X Ver. 1.81 program using the default multiple alignment parameters, and a phylogenetic tree was constructed with MEGA 5.1 using Neighbor-Joining Method.

\section{Embryo Isolation and cDNA Synthesis}

Living sperm cell, egg cell, and embryo isolation were conducted according to the previous procedure (Zhao et al., 2014). To evaluate cell viability, embryos were stained with fluorescein diacetate (FDA). Isolated embryos were incubated in a solution containing $11 \%$ mannitol and $2 \mu \mathrm{g} \mathrm{ml}^{-1}$ FDA for $15 \mathrm{~min}$ at room temperature and washed twice with $11 \%$ mannitol before observation (Zhao et al., 2013). Intact mRNA was directly isolated from embryos using Dynabeads mRNA DIRECT ${ }^{\mathrm{TM}}$ Micro Kit (Life Technologies, USA), and cDNA synthesis and amplification were performed with SMARTer ${ }^{\mathrm{TM}}$ Pico PCR cDNA Synthesis Kit (Clontech, USA).

\section{RNA Isolation and RT-qPCR}

Total RNA of leaf, root, stem, anther, pollen, pollen tube, petal, and sepal were extracted using TRI Reagent Solution (Life technologies, USA), and total RNA of seeds were extracted with RNAqueous ${ }^{\mathrm{TM}}$ (Life technologies, USA). cDNA preparation from total RNA was conducted according to the previous procedure (Zhao et al., 2014). Quantitative real-time reverse transcription-PCR (RT-qPCR) was applied to analyze the expression pattern of WOX family genes. RT-qPCR was performed in a $10 \mu \mathrm{l}$ mixture containing $5 \mu \mathrm{l} 2 \times$ FastStart Essential DNA Green Master (Roche, Germany), $250 \mathrm{nM}$ each primer, and cDNA templates Table S2. RT-qPCR was performed as the following procedure: activation of FastStart TaqDNA polymerase at $95^{\circ} \mathrm{C}$ for $10 \mathrm{~min}$, and 45 cycles $\left(95^{\circ} \mathrm{C}\right.$ for $15 \mathrm{~s}$, annealing at $60^{\circ} \mathrm{C}$ for $20 \mathrm{~s}$; extension at $72^{\circ} \mathrm{C}$ for $30 \mathrm{~s}$ ) on CFXConnect Real-Time system (Bio-Rad, USA). Data processing was performed according to our previous protocol (Ma et al., 2011). Each data represent the mean \pm standard error from three independent experiments.

\section{Subcellular Localization of WOXs}

To investigate the subcellular localization of each WOX, 35S:: YFP-NOS vector was firstly constructed in pCAMBIA1300 to generate $P C A M B I A-35 S:: Y F P-N O S$. Then, each WOX coding sequence (without stop codon) was cloned into the vector pCAMBIA-35S::YFP-NOS to generate 35S::WOX-YFPNOS expression vector. All vectors were transferred into Agrobacterium tumefaciens strain GV3101, and co-expressed with a nucleus marker H2B-CFP in N. benthamiana according to Sparkes (Sparkes et al., 2006). Transformed leaf epidermal cells were observed using a confocal microscope (Leica TCS SP8, Germany).

\section{Transient Transcriptional Activity Analysis}

The reporter vector 35S::GAL4-FLUC-NOS and reference vector 35S::RLUC-NOS was constructed in the pCAMBIA1300, respectively. To generate effector vectors, 35S::GAL4BD-NOS was firstly constructed in PCAMBIA1300 to generate $p C A M B I A-$ 35S::GAL4BD-NOS. Then each WOX coding sequence (without stop codon) was cloned in-frame with GAL4BD into the vector $p$ CAMBIA-35S::GAL4BD-NOS to generate 35S::GAL4BDWOX-NOS expression vector. All vectors were transferred into Agrobacterium tumefaciens strain GV3101 for transient expression. Agrobacterium solutions were then mixed at an $\mathrm{OD}_{600}$ of 0.1 for reporter and effector vectors, and an $\mathrm{OD}_{600}$ of 0.02 for the reference vector. A standard size of the Agrobacterium-infiltrated leaf was collected and ground in $250 \mu \mathrm{l}$ of $1 \times$ Passive Lysis Buffer (Promega, USA) for protein extraction. Relative luciferase activity (FLuc/RLuc) of each effector was analyzed using Dual-Luciferase Reporter Assay (Promega, USA). Transcriptional activity analysis experiment was repeated four times. Each data represents the mean \pm standard error from four independent experiments.

\section{Transcriptional Activation Analysis in Yeast}

For transcriptional activation assay, each WOX coding sequence (without stop codon) was cloned in-frame with GAL4 DNA binding domain in pGBKT7 to construct pGBKT7-WOX. pGBKT7 empty vector was used as a negative control. Different vectors were transformed into yeast strain AH109, respectively. These transformants were inoculated on the SD/Trp- and $\mathrm{SD} /$ Trp-/His-/Ade- medium. After incubated at $28^{\circ} \mathrm{C}$ for 6 days, the growth status of each transformant was checked. This experiment was repeated independently three times.

\section{RESULTS}

\section{Characterization of Early Embryogenesis in Tobacco}

To elucidate the dynamic expression of WOX family genes with a special concern about early events of embryogenesis, N. tabacum, a typical model plant for the study of embryogenesis (He et al., 2007; Zhao et al., 2013, 2016), was chosen for detailed analysis. Division pattern of the zygote and its daughter cells was carefully observed. First asymmetric zygote division leads to the formation of a small apical cell and a larger basal cell as that in A. thaliana (Figures 1A,B). The small apical cell will divide earlier than 

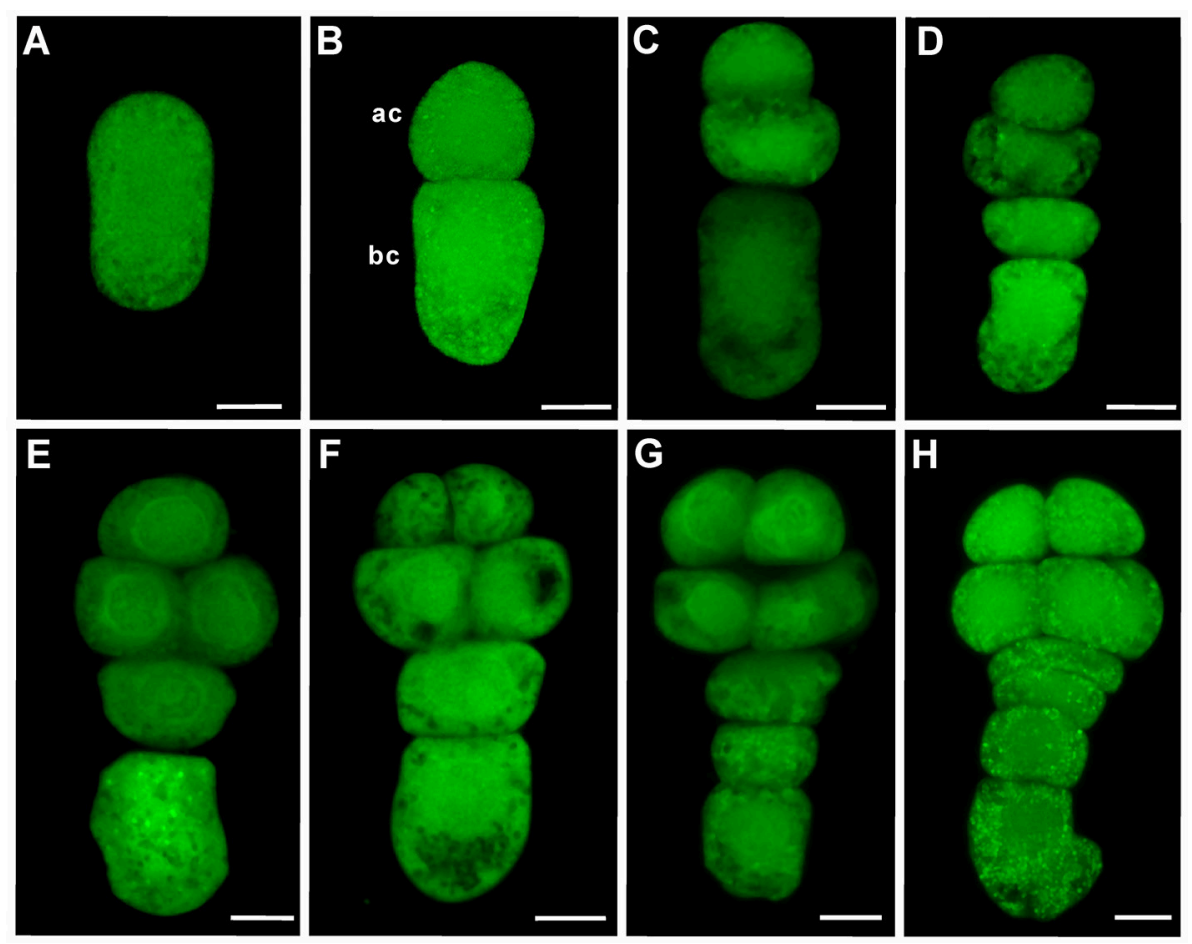

FIGURE 1 | The process of early embryogenesis in tobacco. (A) Elongated zygote, (B) Two-celled proembryo, (C) Three-celled proembryo, (D) Four-celled proembryo, (E) Five-celled proembryo, (F) Six-celled proembryo, (G) Eight-celled embryo with a three-celled suspensor, (H) Eight-celled embryo with a four-celled suspensor. Embryos were stained with FDA. Ac: apical cell; Bc: basal cell. Bar $=10 \mu \mathrm{m}$.

the larger basal cell, giving rise to the formation of a threecelled proembryo (Figure 1C). Later, the larger basal cell divide transversely, leading to the formation of a four-celled proembryo (Figure 1D). Subsequently, two daughter cells of the basal cell divide transversely, giving rise to the formation of a four-celled suspensor at eight-celled embryo stage (Figures 1E-H). These observations confirm that tobacco is an ideal model eudicot for the study of embryogenesis, which displays a highly stereotyped and predictable cell division pattern.

\section{Identification of WOX Family Genes in Tobacco}

To identify WOX family genes in tobacco, a tBLASTn search was carried out using A. thaliana WOX protein sequences in the draft genome sequences, mRNA sequences and EST sequences deposited in NCBI, respectively. Returned sequences related to WOX family genes were collected and assembled. After removing redundant sequences, 13 independent contigs were obtained. cDNA sequence of each contig was further confirmed by RTPCR with gene-specific primes. ORF analysis revealed that each of them has a complete ORF of 555-1149 nucleotides (Table 1). BLASTP searches using their deduced protein sequences in NCBI returned several WOX family proteins from different species, implying that these genes are indeed members of WOX family genes. Thus, they were nominated according to the name of their sequence homology in A. thaliana. There are two striking features of WOX family genes in tobacco genome. The first is that several orthologs to AtWOX6, AtWOX7, AtWOX8, AtWOX10, AtWOX12, and AtWOX14 have not been identified in tobacco genome. The second is that two WOXs (NtWOX3 and NtWOX13) have multiple paralogs derived from chromosomal duplication. To investigate their genomic structures, a blastn search using fulllength cDNA of each WOX was executed in the draft genome sequences. Genomic sequences related to each WOX were collected and compared with their cDNA sequences, respectively. The results revealed that each cDNA has its corresponding genomic sequence, which indicated that these WOX family genes really exist in tobacco genome. In addition, each WOX gene displays a common feature that the exons were separated by introns in the genome (Figure S1). However, the number of introns display substantial variations, varying from one to three. Compared to the conserved exon size (109-560 bp), the length of introns were more divergent, varying from $93 \mathrm{bp}$ to $2 \mathrm{~kb}$ (Figure $\mathrm{S} 1$ ). It is also noteworthy that the highly conserved HD is usually located in the first or the second exon, and not separated by the insertion of introns.

\section{Phylogenetic Analysis of WOXs From Different Species}

To examine the evolutionary relationship of the WOX family genes, the sequences related to WOX family genes from G. max, O. sativa, Z. mays, and S. bicolor were extracted firstly. Due to the lack of consensus nomenclature of WOX family genes, there are various names for WOXs in different publications and databases, 
TABLE 1 | Detailed information for WOX family proteins in tobacco.

\begin{tabular}{|c|c|c|c|c|c|c|c|c|}
\hline \multirow[t]{2}{*}{ Name } & \multirow[t]{2}{*}{ ORF (bp) } & \multicolumn{7}{|c|}{ Predicated protein information } \\
\hline & & $\begin{array}{l}\text { Position of HD } \\
\text { domain }\end{array}$ & $\begin{array}{l}\text { Position } \\
\text { "LFP" }\end{array}$ & $\begin{array}{l}\text { No. of amino } \\
\text { acids }\end{array}$ & $\begin{array}{l}\text { Mol. Wt } \\
\text { (kDa) }\end{array}$ & $\begin{array}{c}\text { isoelectric } \\
\text { point }\end{array}$ & $\begin{array}{l}\text { Signal } \\
\text { peptide }\end{array}$ & $\begin{array}{l}\text { Nuclear location } \\
\text { signal position }\end{array}$ \\
\hline NtWOX1 & 1,149 & 76 & 333 & 383 & 43.4 & 7.2 & - & 136 \\
\hline NtWOX2 & 738 & 15 & 174 & 246 & 28.0 & 8.6 & - & 133 \\
\hline NtWOX3a & 588 & 1 & 182 & 196 & 22.7 & 9.4 & - & - \\
\hline NtWOX3b & 678 & 9 & 195 & 226 & 25.9 & 9.4 & - & - \\
\hline NtWOX3c & 537 & 1 & 165 & 179 & 21.2 & 10.0 & - & - \\
\hline NtWOX3d & 555 & 1 & 164 & 185 & 21.3 & 9.3 & - & - \\
\hline NtWOX4 & 705 & 92 & 225 & 235 & 26.8 & 9.2 & - & 192 \\
\hline NtWOX5 & 549 & 21 & 135 & 183 & 21.0 & 6.9 & - & 81 \\
\hline NtWOX9 & 1,143 & 47 & - & 381 & 42.1 & 7.3 & - & - \\
\hline NtWOX11 & 798 & 20 & 162 & 266 & 28.8 & 5.5 & - & - \\
\hline NtWOX13a & 732 & 88 & - & 244 & 27.6 & 5.4 & - & - \\
\hline NtWOX13b & 810 & 87 & - & 270 & 30.2 & 5.5 & - & - \\
\hline NtWUS & 933 & 47 & 250 & 311 & 35.2 & 6.6 & - & - \\
\hline
\end{tabular}

leading to the confusion for distinguishing the some member of WOX family genes from that in other species. To make less confusing, we nominated each WOX gene derived from different species according to the name of its homology in A. thaliana, which was discovered earliest and explored most extensively (Table S1). Sequence alignment of HD protein sequences from 84 WOXs identified from N. tabacum, A. thaliana, G. max, O. sativa, Z. mays, and S. bicolor were performed as shown in Figure $\mathrm{S} 2$. Twelve residues in HD were completely conserved among all 84 WOXs, indicating a high degree of evolutionary conservation of WOXs. Then, phylogenetic tree based on HD sequences from N. tabacum, A. thaliana, G. max, O. sativa, Z. mays, and $S$. bicolor was constructed using neighbor-joining method. Generally, phylogenetic tree of WOX family proteins could be divided into three clades including WUS clade, intermediate clade and ancient clade (Figure S3). WUS clade is the largest clade composed of 49 WOXs including WUS and WOX1-7 derived from different species. The second subgroup is the intermediate clade composed of WOX8, WOX9, WOX11, and WOX12. The smallest subgroup is the ancient group which only contains three WOXs (WOX10, WOX13, and WOX14). Significant divergence exists in the WOX8/9 branch; no close AtWOX8 relative exists in both monocots and eudicots in the phylogenetic tree, whereas all genomes contain AtWOX9 orthologs. In addition, some other WOXs including (WOX6, WOX7, WOX10, and WOX14) were also lost in tobacco genome. Despite the loss of several individual WOXs, duplication of the tobacco genome is evident as that two tobacco WOX family genes (NtWOX3 and NtWOX13) have multiple paralogs derived from chromosomal duplication within the genome, which has also been found in other plants such as WOX 2 and WOX5 in maize (Figure S3 and Table S1). In addition, the distribution of WOXs is divergent. Nine WOXs fall into the WUS clade, and two WOXs fall into the intermediate (NtWOX9 and NtWOX11) and ancient clade (NtWOX13a and NtWOX13b), respectively.

\section{Characterization of WOX Family Genes in Tobacco}

To characterize WOX family proteins in detail, the basic features and functional motifs of each WOX were investigated. The length of WOX protein sequences in tobacco is divergent, ranging from 183 amino acids for $\mathrm{NtWOX3c}$ to 383 amino acids for NtWOX1, with theoretical molecular weight ranging from 21.0 to $43.4 \mathrm{kDa}$ (Table 1). To identify conserved motifs of WOXs, the protein sequences of 13 WOXs were aligned, and two conserved region motifs have been identified (Figures S4, S5). Generally, the overall structure of WOXs was highly conserved, with an HD located in the N-terminal region, and a WUSbox motif in the $\mathrm{C}$ terminus (Figure S5). Conserved HD is usually composed of 66 residues, which usually fold into a DNAbinding domain. There are 14 positions in HD that are occupied by the same amino acids in tobacco WOXs (Figure S4). The WUS-box motif is another hallmark for WOXs, which could be detected in the carboxy-terminal of most WOXs with three exceptions (NtWOX9, NtWOX13a, and NtWOX13b) (Figure S4). However, the positions of HD and WUS-box motif in the proteins are divergent. The HD domain of most WOX proteins, with three exceptions (NtWOX4, NtWOX13a, and

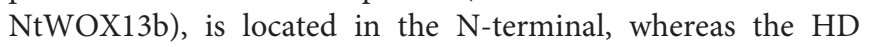
domain of NtWOX4, NtWOX13a, and NtWOX13b are located in the middle of proteins. In contrast to the position of $\mathrm{HD}$ domain, the WUS-box motif of most WOXs except NtWOX11 was found in the same relative position in the carboxy-terminal (Figure S5). Another EAR-like motif (SLELSL), possibly involved in transcriptional repression (Ohta et al., 2001), could only be detected in the C-terminal region of WUS.

WOX is a specific subclade of $\mathrm{HB}$ transcription factor in plants, which is characterized by the presence of HD that could recognize and bind to distinct DNA sequences. In animals, threedimensional structures of homeodomain-DNA complex and the mechanisms of HDs selecting their targeted DNA sequences have 
been comprehensively analyzed. Interactions between $\mathrm{HD}$ and their targeted DNA sequences are achieved by the "recognition" helix in DNA major groove and the $\mathrm{N}$-terminal arm in the minor groove. However, DNA binding sequences of HD in WOXs and the mechanism for recognizing their targets in plants are still largely unknown. To predicate potential DNA targets of WOXs, Clustal X alignment of 72 typical HDs identified in $D$. melanogaster and $13 \mathrm{HDs}$ from tobacco was performed (Figure S6), and three-dimensional structure of HDs was modeled according to the alignment mode as implemented in SWISSMODEL (Figure S7). HDs in animals are usually composed of 60 amino acids that fold into a stable three-helix bundle preceded by a flexible N-terminal arm. Helices II and III are linked by a tight turn, and then give into a helix-tum-helix motif which recognizes their targeted DNA sequences. Homology modeling of HDs revealed that HD of WOXs display a conserved threedimensional structure consisting three helixes connected by two loops (Figure S7). Alignment of HDs from the D. melanogaster and $N$. tabacum revealed that the HDs from $N$. tabacum are usually longer than that in D. melanogaster. Four additional amino acids were found in the second loop between Helices II and III (Figure S6), which could also be detected in WOXs from other plants (Figure S2). However, the role of the four-aminoacid loop in WOXs is still unknown.

\section{Expression Profile of WOXs in Tobacco}

To gain insight into the expression profile of WOXs, RT-PCR was firstly carried out using cDNA template from leaf, stem, root, ovule, and seed. GAPDH was used as the internal reference for PCR. The transcripts of most WOXs with one exception (WOX3d) could be detected in one or more tissues tested (Figure 2A). Interestingly, four WOXs (WOX2, WOX3c, WOX9, and $W U S$ ) display a seed-specific expression pattern. To explore their expression pattern in detail, the relative expression level of each WOX in different tissues were detected through RT-qPCR. Heatmap analysis based on their relative expression levels was carried out, and an overview of the expression pattern of WOXs in tobacco is presented. Most WOXs display a tissue-specific expression pattern, such as WOX2 which could only be detected in seeds. However, some other WOXs (such as WOX11, WOX13a, and WOX13b) display a relatively broad expression pattern, which could be detected in most tissues tested (Figure 2B).

\section{WOXs Display a Stage-Specific Expression Pattern During the Process of Embryogenesis}

Expression pattern analysis of WOXs in A. thaliana revealed an outstanding expression characteristics of WOXs during embryo development: cell type-specific and stage-specific expression programs. In A. thaliana, it was reported that WOX9 expression was initiated in the basal cell and subsequently shifted into embryo proper. WOX5 which marks the quiescent center was initiated in the hypophysis cell at around 32-celled embryo stage. The transcripts of WUS was detected as early as in 16celled embryo stage and confined to the four inner cells of the apical region (Mayer et al., 1998; Haecker et al., 2004). However, whether WOXs in other plants display a conserved expression pattern in early embryogenesis is still largely unknown. In the present work, cDNA from embryos at successive stages (from stage 1 to stage 9) were prepared for RT-qPCR according to our previous protocol (Zhao et al., 2013). Generally, 10 WOXs could be detected in embryos at one or more stages. Similar to WOXs in A. thaliana, WOXs in tobacco display a stage-specific expression pattern during the process of embryogenesis (Figure 3). The transcripts of five WOXs (WOX2, WOX9, WOX11, WOX13a, and $W O X 13 b$ ) could be detected in embryos as early as stage 1 (2-celled proembryo stage) and presented in the whole process of embryo development (Figure 3). WUS and WOX5 initiated their transcription in stage 3 (8-celled embryo stage) and stage 4 (32-celled embryo stage), respectively. WOX 1 initiated its transcription in the middle stages of embryo development (stage 5). WOX3c and WOX3d initiated their transcription much later than other WOX family genes. Their transcripts could only be detected in stage 8 (heart-shaped embryo stage) and stage 9 (torpedo-shaped embryo stage), respectively (Figure 3).

\section{Parental-of-Origin Transcripts and de Novo Transcripts of WOXs Are Confirmed in Zygote}

Unlike animals, in which zygote genome is quiescent and maternally derived factors deposited in egg cell are enough to drive early embryo development, plant zygotes are transcriptionally active and begin to transcribe to generate transcripts for early embryo development (Ning et al., 2006; Zhao et al., 2011, 2017; Del Toro-De Leon et al., 2014). Early expression pattern analysis of WOXs in A. thaliana revealed that WOX2 and WOX 8 were co-expressed in the zygote, and WOX9 initiated its transcription in the basal cell (Haecker et al., 2004). Recently, both paternal factors SSP and maternal factors HDG11/12 were reported to regulate the expression of WOX8 in the zygote, which is critical for embryo pattern formation (Ueda et al., 2017). Whether WOX transcripts in zygote is delivered by gametes or de novo transcribed is not clear. To answer this question and test whether the expression of WOXs in gametes and early embryos are similar to that in A. thaliana, the relative expression level of WOXs in sperm cells, egg cells, zygotes, and 2-celled proembryos were quantified (Figure 4). Unlike A. thaliana, the transcripts of WOX2 was firstly detected in zygotes, but not in egg cells and sperm cells (Figure 4). WOX9 displays a similar expression pattern as WOX2, which initiated its transcription as early as in zygote, indicating their de novo transcription rightly after fertilization. More interestingly, both WOX11 and WOX13b show high expression levels in egg cells, but decrease significantly in zygotes. This specific expression pattern of WOX11 and WOX13b suggests that their transcripts in zygote are likely delivered by egg cells.

\section{Uneven Expression of WOXs in Apical and Basal Cell Lineages}

First asymmetric zygote division is one of the critical cell divisions in early embryogenesis, which usually gives rise to the formation of two daughter cells with different division pattern 
A

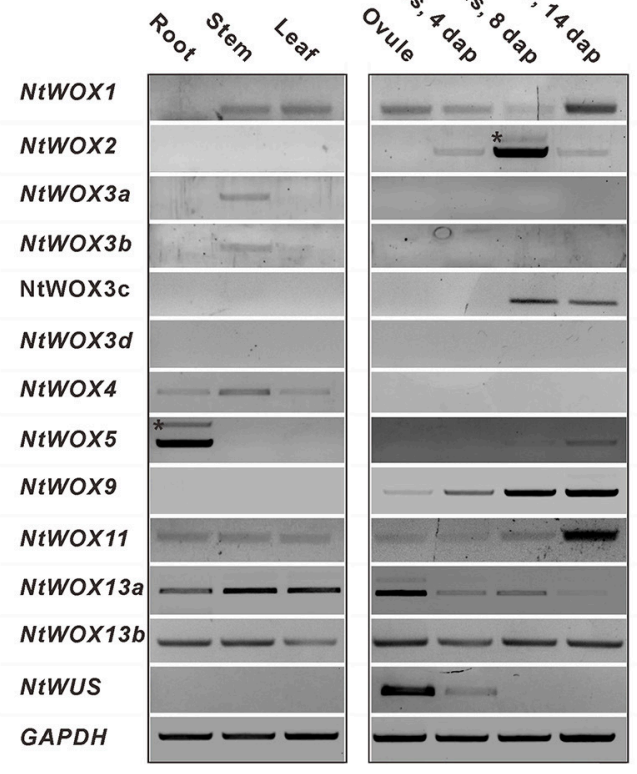

B

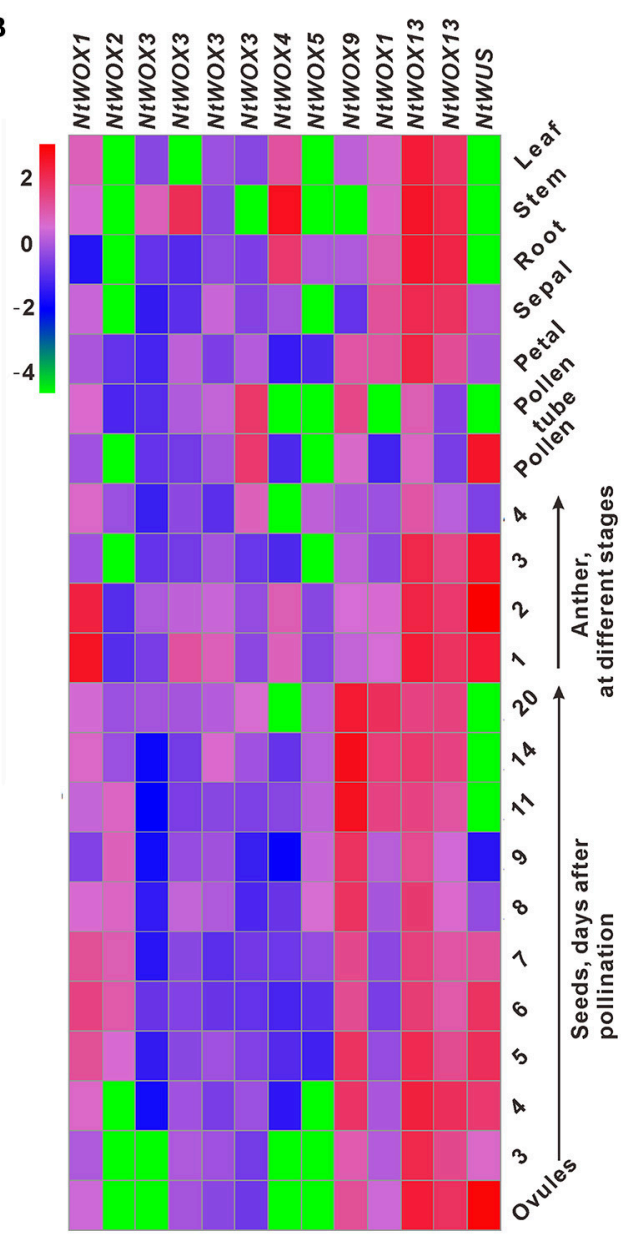

FIGURE 2 | Expression pattern analysis of WOXs in tobacco. (A) RT-PCR analysis of the expression of WOXs. cDNA prepared from root, stem, leaf, ovule, and seeds at different stages were selected as templates for PCR. GAPDH was used as the control. * indicate no specific bands. (B) Expression profile of WOXs in tobacco, which was constructed based on the relative expression level of WOXs in different tissues. The expression level was normalized to the average expression level of GAPDH (AJ133422), polyubiquitin (GQ281244). Each data represents the mean of three biological repeats. A red box indicates a higher expression level, whereas a green box indicates the lower expression level. Anthers at stages 1-4 correspond to anthers containing microspore mother cells, tetrads, single-nucleated pollen, and bi-nucleated pollen, respectively. The scale bar represents the fold change (log10 value).

and distinct developmental fates. Previous transcription profile analysis revealed that asymmetric zygote division resulted in uneven distribution of some specific transcripts in apical and basal cell, respectively (Ma et al., 2011). Similar transcription profile analysis of apical and basal cell has not been performed in Arabidopsis, possibly due to the difficulty in isolating small living apical cell and basal cell. However, expression pattern analyzed by in situ hybridization revealed that different WOXs display cell type-specific expression pattern. WOX2 and WOX8 were found to be confined to the apical cell and basal cell after zygote division, respectively. WOX9 was firstly detected in the basal cell and subsequently shifted to the descendants of the apical cell (Haecker et al., 2004). To test whether WOXs also show a similar pattern in apical and basal cell lineages of early embryo in tobacco, apical cell and basal cell linages of 2-celled proembryo (Figure 5A) and around 32-celled embryo (Figure 5B) were isolated for mRNA extraction and cDNA synthesis, respectively.
RT-qPCR was used to compare their relative expression levels in apical and basal cell linages. Surprisingly, none of them are exclusively expressed in apical cell or basal cell. The transcripts of WOX 2 and WOX9 could be detected in both apical and basal cell. However, WOX2 show a significant higher expression level in apical cell compared with that in basal cell ( $>8$-fold), and become confined to embryo proper at 32-celled embryo stage. WOX9 show a significant higher expression level in basal cell ( $>2$-fold compared with that in apical cell) and suspensor cells ( $>20$-fold compared with that in embryo proper), indicating that the transcription of WOX 2 and WOX 9 was gradually restricted to the apical or basal cell linages as the embryo develop, respectively. More interestingly, the expression of WOX11 was also confined to embryo proper at 32-celled embryo stage, and WUS initiated its transcription specifically in embryo proper (Figure 5). Thus, despite no specific expression of WOX family genes in apical or basal cell, the expression pattern of WOXs in apical and basal cell 

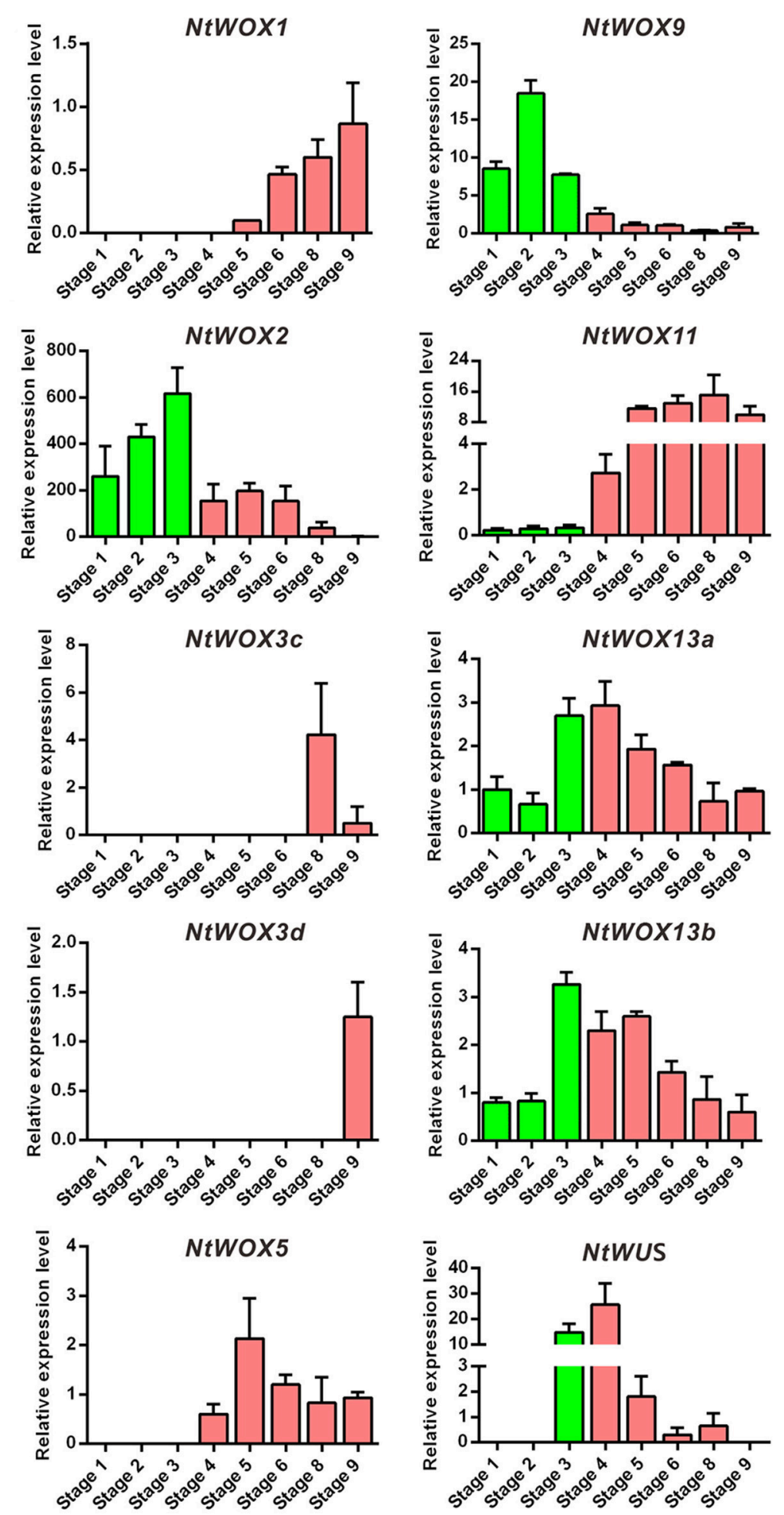

FIGURE 3 | Stage-specific expression pattern of WOXs during the process of embryogenesis. The expression level of each WOX in embryo at stages 9 (except WUS) was set as 1. The expression level was normalized to the average expression level of GAPDH (AJ133422), polyubiquitin (GQ281244), and elongation factor 1 $\alpha$ (AF120093). Error bars represent mean \pm standard error from three independent experiments. Nine successive stages of embryogenesis were classified in tobacco as described previously (Zhao et al., 2013). 

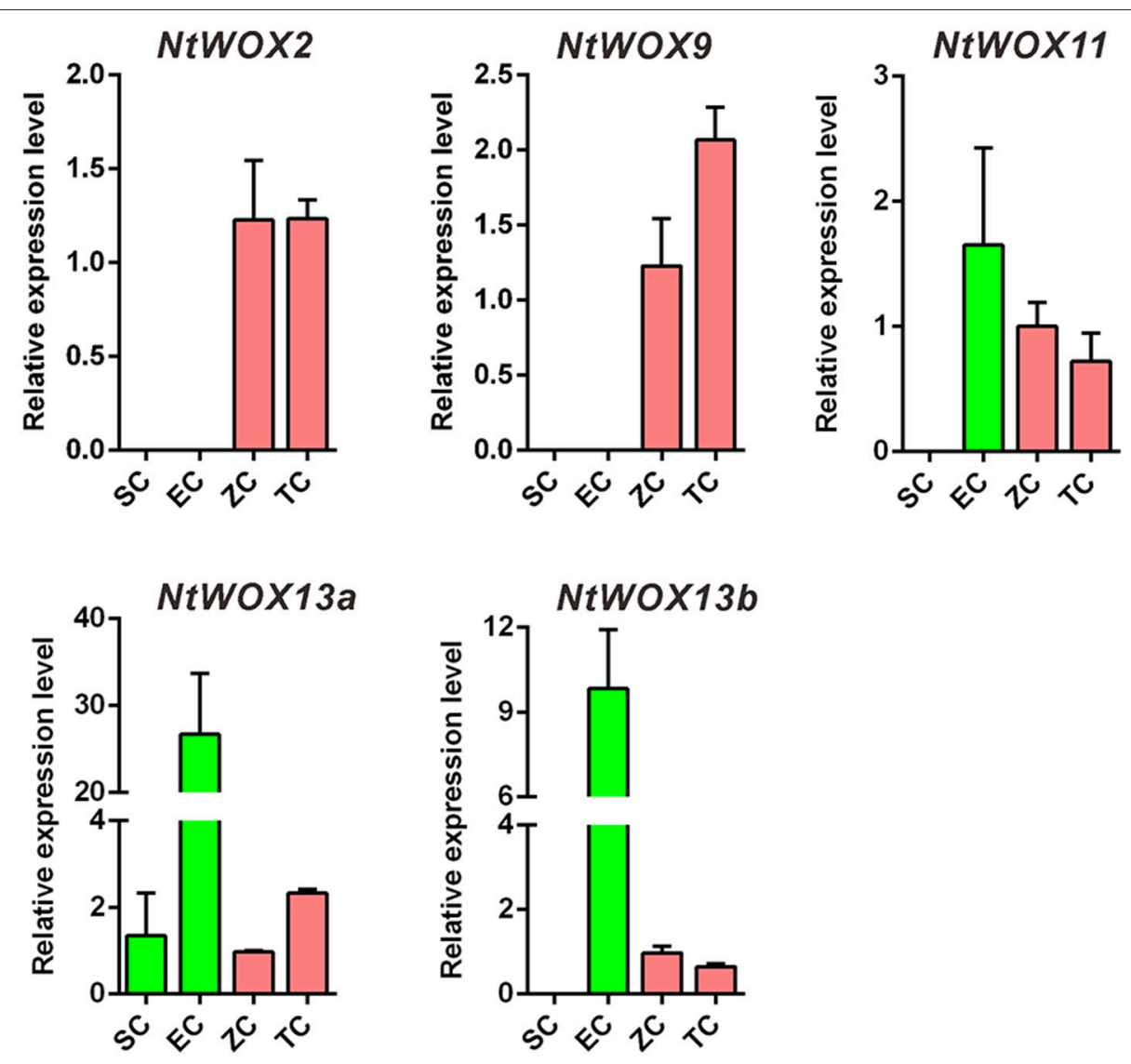

FIGURE 4 | Test of de novo or gamete-delivered transcripts in zygote. Relative expression levels of WOXs in sperm cells (SC), egg cells (EC), zygote (ZC), and 2-celled proembryos (TC). The expression level of each WOX in zygote was set as 1. The expression level was normalized to the average expression level of GAPDH (AJ133422), polyubiquitin (GQ281244), and elongation factor $1 \alpha$ (AF120093). Error bars represent \pm standard error from three independent experiments.

linages of around 32-celled embryo implies a relatively conserved mechanism for apical-basal embryo polarity establishment in both N. tabacum and A. thaliana.

\section{WUS-Box Is an Evolutionarily Conserved Motif for the Repressive Activity of WOXs}

Early reports in A. thaliana revealed that WUS had the strong repressive activity to suppress the expression of the reporter gene due to its WUS box (Ikeda et al., 2009). Recently, research about Medicago truncatula WOX gene, STENOFOLIA (STF), further confirmed that transcriptional repressor activity of STF was conferred by WUS box in the C-terminal (Lin et al., 2013). However, the transcriptional activities of all WOX member in a certain species have not been determined. And whether the role of WUS box for transcriptional repressor activity is conserved among different plants is still largely unknown. To determine which WOXs in tobacco acts as a transcriptional activator or repressor, the intracellular localization of each WOX was firstly investigated. The construct 35S::WOX-YFP for each WOX was generated respectively. And the construct 35S::YFP was chosen as a control. The results revealed that all WOXs could be detected in the nucleus, corresponding to the characteristics of transcriptional factors (Figure 6).

Next, we examined their transcriptional activities through luciferase transient expression assays in leaf epidermis of $N$. benthamiana. The effector plasmid contained each WOX fused with GAL4 DNA-binding domain, and the reporter plasmid contained a $5 \times$ GAL4-binding site fused with the luciferase (LUC) gene (Figure 7A). Bioluminescence measurements revealed all WOXs in WUS clade could reduce luciferase activities ( $>2$-fold), indicating strong repressive activity of these WOXs. However, four WOXs (WOX9 and WOX11 in the intermediate clade, WOX13a and WOX13b in ancient clade) led to the significant increase of luciferase activities, indicating strong activation activities of these four WOXs (Figure 7B). By comparison of these WOX protein sequences, we found these four WOX proteins lack typical WUS-box motif "TLXLFPXX," suggesting that WUS-box in WOX proteins are likely responsible for their repressive activities. More interestingly, WOX11 in intermediate clade with a WUS box-like motif "TNXLFPXX" (with a substitution in the second position of the motif) in the upstream of typical WUS box also displayed no ability to repress the luciferase activities (Figure S5), suggesting that 


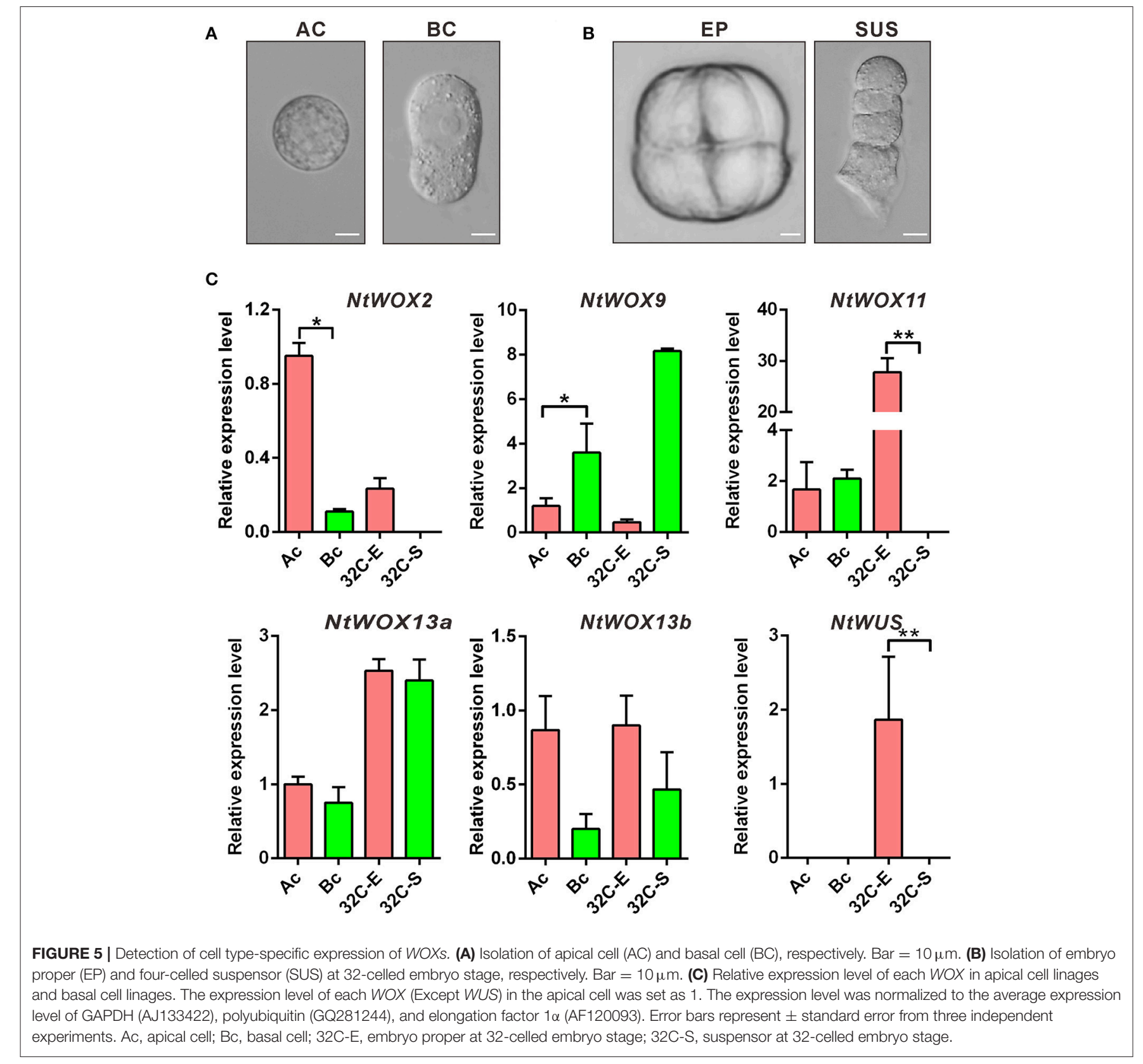

the core amino acids of WUS box or the position of WUS box-like motif in WOX protein is critical for the repressive activity of WOXs (Figure S5). To confirm this result, four WOXs with activation abilities were fused to the GAL4 DNA-binding domain in pGBKT7 and transformed into yeast strain to test their abilities for transcriptional activation. Consistent with the result of luciferase transient expression assay, these four WOXs can activate the expression of reporter genes in yeast (Figure 7C). These results suggest that the original WOXs have transcriptional activation capacity, whereas repressive ability was equipped later through the acquisition of WUS-box as a repressive motif in the $\mathrm{C}$-terminal of the protein. Consistent with this hypothesis, no WUS-box motif could be detected in ancient WOXs from unicellular green algae Ostreococcus lucimarinus and Ostreococcus tauri, moss Physcomitrella patens and spikemoss Selaginella moellendorffi (Figure S8). However, which motif contribute to the transcriptional activation ability of WOXs in ancient and intermediate clade is still unknown.

\section{DISCUSSION}

\section{Main Characteristics of WOX Family Genes in Tobacco}

WUSCHEL-related homeobox genes, a plant-specific clade of homeobox transcription factors, have been identified in plants 


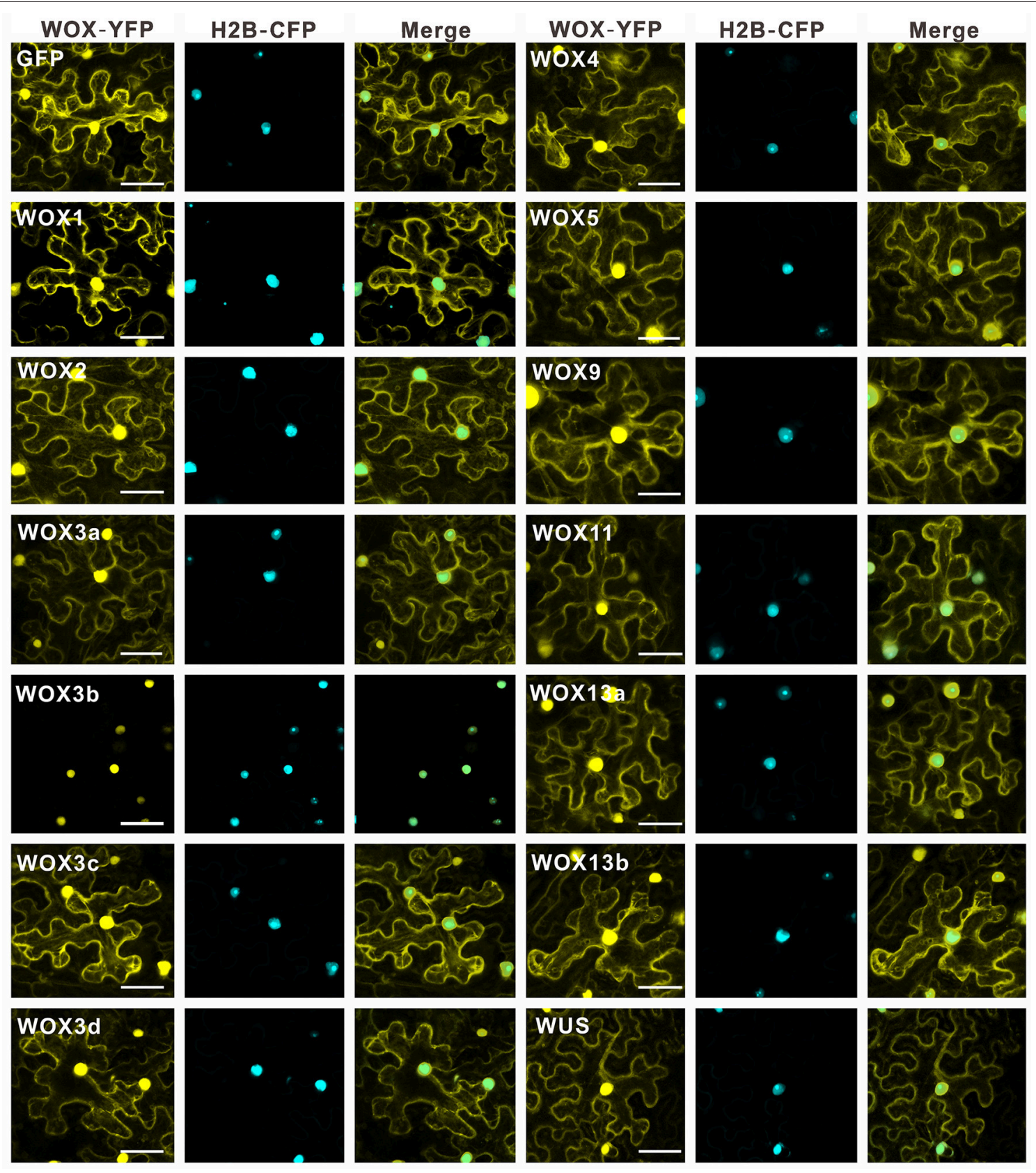

FIGURE 6 | Subcellular location analysis of WOX family proteins in N. benthamiana leaves. YFP alone was used as a control. Bar $=50 \mu \mathrm{m}$.

from different clans (Mukherjee et al., 2009). Researches revealed that WOXs in A. thaliana have important roles in different developmental processes, especially in embryo development including cell fate determination of apical and basal cell lineages and the establishment of the apical-basal pattern (Haecker et al., 2004; Breuninger et al., 2008). However, variations in their expression pattern and roles among different plant species are still largely unknown. $N$. tabacum has long been considered as an ideal model plant for embryogenesis, which displays a highly stereotyped and predictable cell division pattern, beginning from the first asymmetric zygote division to embryo maturation. Furthermore, unlike in A. thaliana, relevant techniques for the isolation of gametes and early embryos in tobacco have been wellestablished, which greatly facilitate the investigations of detailed expression pattern of WOXs (He et al., 2007; Zhao et al., 2013, 2016). In this work, 13 WOXs have been identified and could be divided into three groups based on phylogenetic analysis. Phylogenetic analysis revealed significant differences not only in WOXs between eudicots and monocots clade, but also in eudicots clade. First divergence is that no AtWOX8 orthologs was found 

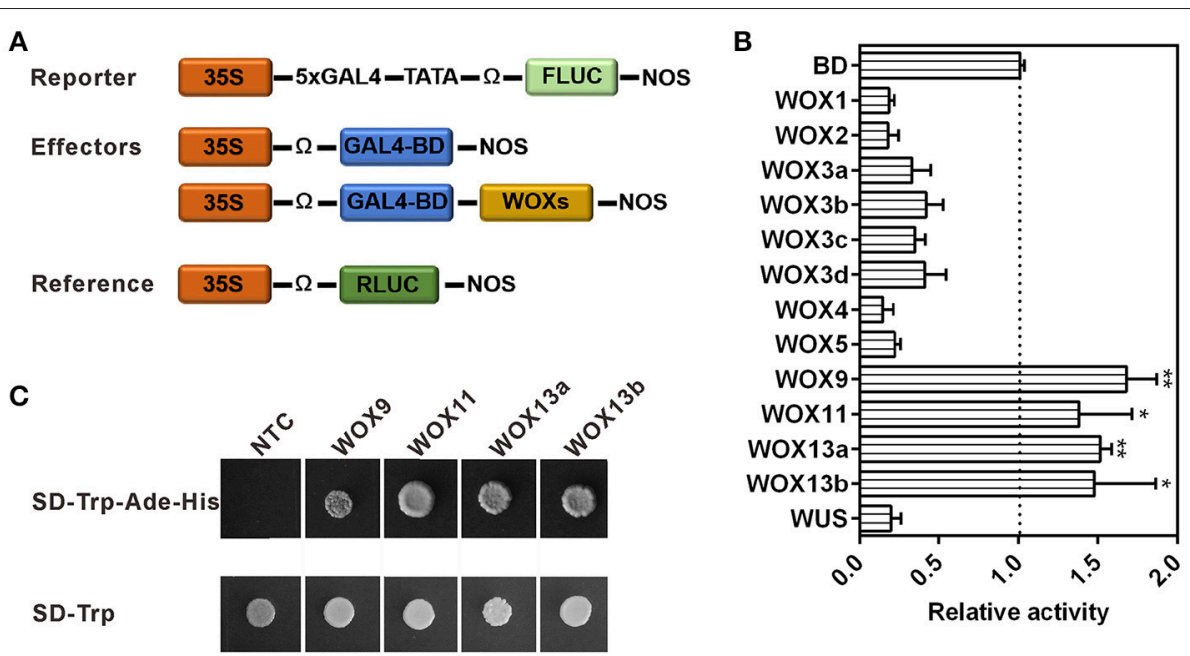

FIGURE 7 | The repressive or activation activities of WOXs in tobacco. (A) Reporter, effector, and reference constructs used in transient expression analysis. (B) Relative luciferase activities of each effector compared with GAL4-DB control. Error bars indicate standard error from four independent experiments. * and ** indicate statistical difference compared to the control ( $t$-test, $p<0.05$, or $p<0.01$, respectively). (C) The transcriptional activation activities of WOXs in yeast.

in monocots and eudicots except $A$. thaliana. In addition, there is no evidence that monocots clade contain orthologs of WOX 1,6 , 7, 10, and 14. Surprisingly, the majority orthologs (except WOX1) that disappeared in monocots clade are also lost in other eudicots, suggesting that $A$. thaliana genome generate some speciesspecific WOXs during the course of the evolution. Another significant divergence is that two tobacco WOXs (NtWOX3 and NtWOX13) have multiple paralogs derived from chromosomal duplication. Duplicated WOXs have also been found in other plants such as WOX2 and WOX5 in maize (Figure S3 and Table S1). Comparable analysis of these WOXs indicated the composition of protein domain and transcriptional activities within each class are similar, suggesting possible functional redundancy of WOXs in same group (Figure S4 and Figure 7).

\section{Structure Base of WOXs for Regulating the Transcription of Their Targets}

The function of WOXs in relation to their specific structure has been discussed in previous works. Among them, WUS, the founding member of WOX family, was approved to mainly act as a repressor of transcription in the maintenance of stem cell identity, but also act as a direct activator of $A G$ gene in flowers (Lohmann et al., 2001). The transcriptional repressive activity of WUS was conferred by the WUS-box in the C-terminal. Similar transcriptional repressor activity has also been found in M. truncatula WOX gene, STF (Lin et al., 2013). However, the relationship between transcriptional activities of WOXs and their protein characteristics has not been comprehensively analyzed. Here, nine of 13 tobacco WOXs have obvious transcriptional repressive activities, whereas other four WOXs display strong activation activities. By comparison of nine WOX protein sequences, a typical WUS-box motif "TLXLFPXX" was identified in the C-terminal of them. Whereas, other four WOXs with transcriptional activation activities lack typical WUS-box motif, suggesting that WUS-box in tobacco WOXs are likely responsible for their repressive activities. More interestingly, phylogenetic analysis results revealed that all nine WOXs with transcriptional repressive activities fall into WUS clade, whereas WOXs with transcriptional activation activities fall into ancient clade or intermediate clade, suggesting that the original WOXs likely act as transcriptional activation factors, whereas transcriptional repressive ability was equipped later through the acquisition of functional WUS-box motif in the process of WOX evolution.

$\mathrm{HD}$ in WOXs is an evolutionarily conserved DNA-binding domain, which is composed of $\sim 60$ amino acids that fold into a flexible $\mathrm{N}$-terminal arm and a stable three-helix bundle. Researches about HOX genes in animals revealed that the DNA binding site of HD is usually formed by the N-terminal arm in the minor groove and a single "recognition" helix in the major groove (Noyes et al., 2008). Despite a common DNA-binding structure, amino acid variations in HD have also been found. Residues both in N-terminal arms and recognition helix contributed to their DNA-binding specificity. Relationship between binding specificities and amino acid sequence in DNAbinding architecture of 84 independent homeodomains from D. melanogaster have been comprehensively analyzed, allowing the prediction of preferred recognition sites of different HOXs from other species (Noyes et al., 2008). However, whether HDs in WOXs display a similar DNA-binding specificity attributed by amino acid sequence in DNA-binding architecture are largely unknown. Homology modeling of HDs revealed that $\mathrm{HD}$ in tobacco WOXs display a relatively conserved structure (Figure S7). Alignment of these HDs with that from $D$. melanogaster revealed a relatively conserved amino residues on $\mathrm{N}$-terminal arm and recognition helix, suggesting a potential conserved mechanism of HDs in WOXs recognizing their targeted DNA sequences. Although similar researches on the analysis of HD specificities have not been performed in plants, the "TTAATGG" motif has been recognized by different WOXs 
from different plants. In A. thaliana, WUS could bind to the "TTAATGG" motif in $A G$ and $R R$ genes (Lohmann et al., 2001; Leibfried et al., 2005). In rice, WOX family proteins $\mathrm{QHB}, \mathrm{WOX} 3$, and WOX11 have also been shown to be able to interact with the same DNA sequence in different targets (Kamiya et al., 2003; Dai et al., 2007; Zhao et al., 2009). Taken together, "TTAATGG" seems to be a consensus binding motif for different WOXs. However, whether different WOX members could recognize other DNA motifs and display recognition specificities as shown in different HDs from $D$. melanogaster are largely unknown. Hence, comparison of recognition specificities conferred by HDs in different WOXs might be a charming work for understanding molecular mechanism of WOXs in the further study.

\section{Commonalities and Differences in the Expression Pattern of WOXs During Early Embryogenesis}

In A. thaliana, WOX family genes are well-known for their specific expression pattern in early embryogenesis. WOX2 became confined to the apical cell linages, whereas WOX8 was restricted in the basal cell linages after zygote division (Haecker et al., 2004). WUS and WOX5 were found to be exclusively located in SAM and RAM after around 32-celled embryo stage, respectively. Similar expression pattern of WUS and WOX5 in SAM and RAM was also found in maize (Nardmann and Werr, 2006; Nardmann et al., 2007). Consistent with this observation, WOX5 initiated its transcription in suspensor at 8-celled embryo stage, and WUS was firstly detected in embryo proper at 8celled embryo stage, suggesting a relatively conserved molecular mechanism for SAM and RAM establishment in both monocots and eudicots. However, some variations in the expression pattern of WOXs between monocots and eudicots have also been found, especially about WOX 2 and WOX 8 at 2-celled proembryo stage. The first striking difference is that no WOX8 has been identified in the genomes of monocots and other eudicots to date. The second is that delayed WOX2 expression was found in monocots (Nardmann and Werr, 2006; Nardmann et al., 2007; Zhao et al., 2017). Consistent with preceding observations, no homolog of WOX 8 has been identified in tobacco genome. On the other hand, the transcripts of WOX 2 could be detected in both the apical cell and basal cell simultaneously, unlike that in A. thaliana. However, the expression of WOX2 in apical cell is significantly higher than that in the basal cell ( $>8$-fold), and became restricted in the embryo proper at the 32-celled embryo stage. All these results implied that the expression pattern of WOX family genes has undergone modifications in the course of evolution, not only in the divergence of eudicots and monocots, but also in different eudicots.

\section{REFERENCES}

Arnold, K., Bordoli, L., Kopp, J., and Schwede, T. (2006). The SWISS-MODEL workspace: a web-based environment for protein structure homology modelling. Bioinformatics 22, 195-201. doi: 10.1093/bioinformatics/b ti770
According to the works in A. thaliana, WOXs such as WOX 2, WOX 8, and WOX 9 play essential roles in cell fate specification and early embryo patterning. Then, an interesting question raises, are these mechanisms initiated and directed by parental instructions or de novo established after fertilization? To answer the question it is necessary to understand the expression behavior of these WOXs before and after fertilization. In the present work, the transcripts of WOX2 and WOX 9 was only detected in the zygotes, but not in egg cells or sperm cells, suggesting that they are de novo transcribed in the zygote, and the relevant mechanism is fertilization-initiated. Interestingly, WOX11 and WOX13b were detected only in the egg cell, but not in the sperm cell, indicating at least in early zygote they show parent-of-origin characteristics. How these maternal transcripts act in early zygote development or initiation of embryogenesis will deserve extensive investigations. Taken together, these WOXs may function successively during the transition from gametophytic to the sporophytic generation and link the developmental signaling from parents to offspring.

\section{ACCESSION NUMBERS}

Sequence data for WOX family genes in tobacco has been deposited in GenBank (http://www.ncbi.nlm.nih.gov/ Genbank) under the following accession numbers: NtWOX1 (MG843879), NtWOX2 (MG843880), NtWOX3a (MG843881), NtWOX3b (MG843882), NtWOX3c (MG843883), NtWOX3d (MG843884), NtWOX4 (MG843885), NtWOX5 (MG843886), NtWOX9 (MG843887), NtWOX11 (MG843888), NtWOX13a (MG843889), NtWOX13b (MG843890), NtWUS (MG843891).

\section{AUTHOR CONTRIBUTIONS}

Conceived and designed the experiments: PZ and MS. Performed the experiments: XZ, YG, and PZ. Analyzed the data: XZ, YG, and PZ. Wrote the paper: PZ and MS.

\section{ACKNOWLEDGMENTS}

This project was supported by National Natural Science Fund of China $(31400171 ; 31600244)$.

\section{SUPPLEMENTARY MATERIAL}

The Supplementary Material for this article can be found online at: https://www.frontiersin.org/articles/10.3389/fpls.2018. 00311/full\#supplementary-material

Breuninger, H., Rikirsch, E., Hermann, M., Ueda, M., and Laux, T. (2008). Differential expression of WOX genes mediates apical-basal axis formation in the Arabidopsis embryo. Dev. Cell 14, 867-876. doi: 10.1016/j.devcel.2008.03.008

Dai, M., Hu, Y., Zhao, Y., Liu, H., and Zhou, D. X. (2007). A WUSCHEL-LIKE HOMEOBOX gene represses a YABBY gene expression required for 
rice leaf development. Plant Physiol. 144, 380-390. doi: 10.1104/pp.107.0 95737

Del Toro-De Leon, G., Garcia-Aguilar, M., and Gillmor, C. S. (2014). Nonequivalent contributions of maternal and paternal genomes to early plant embryogenesis. Nature 514, 624-627. doi: 10.1038/nature13620

Forzani, C., Aichinger, E., Sornay, E., Willemsen, V., Laux, T., Dewitte, W., et al. (2014). WOX5 suppresses CYCLIN D activity to establish quiescence at the center of the root stem cell niche. Curr. Biol. 24, 1939-1944. doi: 10.1016/j.cub.2014.07.019

Gehring, W. J., Affolter, M., and Burglin, T. (1994). Homeodomain proteins. Annu. Rev. Biochem. 63, 487-526. doi: 10.1146/annurev.bi.63.070194.002415

Haecker, A., Gross-Hardt, R., Geiges, B., Sarkar, A., Breuninger, H., Herrmann, M., et al. (2004). Expression dynamics of WOX genes mark cell fate decisions during early embryonic patterning in Arabidopsis thaliana. Development 131, 657-668. doi: 10.1242/dev.00963

He, Y. C., He, Y. Q., Qu, L. H., Sun, M. X., and Yang, H. Y. (2007). Tobacco zygotic embryogenesis in vitro: the original cell wall of the zygote is essential for maintenance of cell polarity, the apical-basal axis and typical suspensor formation. Plant J. 49, 515-527. doi: 10.1111/j.1365-313X.2006.02970.x

Ikeda, M., Mitsuda, N., and Ohme-Takagi, M. (2009). Arabidopsis WUSCHEL is a bifunctional transcription factor that acts as a repressor in stem cell regulation and as an activator in floral patterning. Plant Cell 21, 3493-3505. doi: 10.1105/tpc.109.069997

Jain, M., Tyagi, A. K., and Khurana, J. P. (2008). Genome-wide identification, classification, evolutionary expansion and expression analyses of homeobox genes in rice. FEBS J. 275, 2845-2861. doi: 10.1111/j.1742-4658.2008.06424.x

Kamiya, N., Nagasaki, H., Morikami, A., Sato, Y., and Matsuoka, M. (2003). Isolation and characterization of a rice WUSCHEL-type homeobox gene that is specifically expressed in the central cells of a quiescent center in the root apical meristem. Plant J. 35, 429-441. doi: 10.1046/j.1365-313X.2003.01816.x

Kiefer, F., Arnold, K., Kunzli, M., Bordoli, L., and Schwede, T. (2009). The SWISS-MODEL repository and associated resources. Nucleic Acids Res. 37, D387-D392. doi: 10.1093/nar/gkn750

Leibfried, A., To, J. P., Busch, W., Stehling, S., Kehle, A., Demar, M., et al. (2005). WUSCHEL controls meristem function by direct regulation of cytokinin-inducible response regulators. Nature 438, 1172-1175. doi: 10.1038/nature04270

Lenhard, M., Bohnert, A., Jurgens, G., and Laux, T. (2001). Termination of stem cell maintenance in Arabidopsis floral meristems by interactions between WUSCHEL and AGAMOUS. Cell 105, 805-814. doi: 10.1016/S0092-8674(01)00390-7

Lin, H., Niu, L., Mchale, N. A., Ohme-Takagi, M., Mysore, K. S., and Tadege, M. (2013). Evolutionarily conserved repressive activity of WOX proteins mediates leaf blade outgrowth and floral organ development in plants. Proc. Natl. Acad. Sci. U.S.A. 110, 366-371. doi: 10.1073/pnas.1215376110

Lohmann, J. U., Hong, R. L., Hobe, M., Busch, M. A., Parcy, F., Simon, R., et al. (2001). A molecular link between stem cell regulation and floral patterning in Arabidopsis. Cell 105, 793-803. doi: 10.1016/S0092-8674(01)00384-1

Ma, L., Xin, H., Qu, L., Zhao, J., Yang, L., Zhao, P., et al. (2011). Transcription profile analysis reveals that zygotic division results in uneven distribution of specific transcripts in apical/basal cells of tobacco. PLoS ONE 6:e15971. doi: 10.1371/journal.pone.0015971

Mayer, K. F., Schoof, H., Haecker, A., Lenhard, M., Jurgens, G., and Laux, T. (1998). Role of WUSCHEL in regulating stem cell fate in the Arabidopsis shoot meristem. Cell 95, 805-815. doi: 10.1016/S0092-8674(00)81 703-1

Mukherjee, K., Brocchieri, L., and Burglin, T. R. (2009). A comprehensive classification and evolutionary analysis of plant homeobox genes. Mol. Biol. Evol. 26, 2775-2794. doi: 10.1093/molbev/msp201

Nardmann, J., and Werr, W. (2006). The shoot stem cell niche in angiosperms: expression patterns of WUS orthologues in rice and maize imply major modifications in the course of mono- and dicot evolution. Mol. Biol. Evol. 23, 2492-2504. doi: 10.1093/molbev/msl125

Nardmann, J., Zimmermann, R., Durantini, D., Kranz, E., and Werr, W. (2007). WOX gene phylogeny in Poaceae: a comparative approach addressing leaf and embryo development. Mol. Biol. Evol. 24, 2474-2484. doi: 10.1093/molbev/msm182

Ning, J., Peng, X. B., Qu, L. H., Xin, H. P., Yan, T. T., and Sun, M. (2006). Differential gene expression in egg cells and zygotes suggests that the transcriptome is restructed before the first zygotic division in tobacco. FEBS Lett. 580, 1747-1752. doi: 10.1016/j.febslet.2006.02.028

Noyes, M. B., Christensen, R. G., Wakabayashi, A., Stormo, G. D., Brodsky, M. H., and Wolfe, S. A. (2008). Analysis of homeodomain specificities allows the family-wide prediction of preferred recognition sites. Cell 133, 1277-1289. doi: 10.1016/j.cell.2008.05.023

Ohta, M., Matsui, K., Hiratsu, K., Shinshi, H., and Ohme-Takagi, M. (2001). Repression domains of class II ERF transcriptional repressors share an essential motif for active repression. Plant Cell 13, 1959-1968. doi: 10.1105/tpc.13.8.1959

Pearson, J. C., Lemons, D., and Mcginnis, W. (2005). Modulating Hox gene functions during animal body patterning. Nat. Rev. Genet. 6, 893-904. doi: $10.1038 / \operatorname{nrg} 1726$

Petersen, T. N., Brunak, S., Von Heijne, G., and Nielsen, H. (2011). SignalP 4.0: discriminating signal peptides from transmembrane regions. Nat. Methods 8 , 785-786. doi: 10.1038/nmeth.1701

Schoof, H., Lenhard, M., Haecker, A., Mayer, K. F., Jurgens, G., and Laux, T. (2000). The stem cell population of Arabidopsis shoot meristems in maintained by a regulatory loop between the CLAVATA and WUSCHEL genes. Cell 100, 635-644. doi: 10.1016/S0092-8674(00)80700-X

Sparkes, I. A., Runions, J., Kearns, A., and Hawes, C. (2006). Rapid, transient expression of fluorescent fusion proteins in tobacco plants and generation of stably transformed plants. Nat. Protoc. 1, 2019-2025. doi: $10.1038 /$ nprot.2006.286

Ueda, M., Aichinger, E., Gong, W., Groot, E., Verstraeten, I., Vu, L. D., et al. (2017). Transcriptional integration of paternal and maternal factors in the Arabidopsis zygote. Genes Dev. 31, 617-627. doi: 10.1101/gad.292409.116

van der Graaff, E., Laux, T., and Rensing, S. A. (2009). The WUS homeobox-containing (WOX) protein family. Genome Biol. 10:248. doi: 10.1186/gb-2009-10-12-248

Vollbrecht, E., Veit, B., Sinha, N., and Hake, S. (1991). The developmental gene Knotted-1 is a member of a maize homeobox gene family. Nature 350, 241-243. doi: 10.1038/350241a0

Zhao, J., Xin, H., Cao, L., Huang, X., Shi, C., Zhao, P., et al. (2016). NtDRP is necessary for accurate zygotic division orientation and differentiation of basal cell lineage toward suspensor formation. New Phytol. 212, 598-612. doi: 10.1111/nph.14060

Zhao, J., Xin, H., Qu, L., Ning, J., Peng, X., Yan, T., et al. (2011). Dynamic changes of transcript profiles after fertilization are associated with de novo transcription and maternal elimination in tobacco zygote, and mark the onset of the maternal-to-zygotic transition. Plant J. 65, 131-145. doi: 10.1111/j.1365-313X.2010.04403.x

Zhao, P., Begcy, K., Dresselhaus, T., and Sun, M. X. (2017). Does early embryogenesis in eudicots and monocots involve the same mechanism and molecular players? Plant Physiol. 173, 130-142. doi: 10.1104/pp.16.01406

Zhao, P., Zhou, X. M., Zhang, L. Y., Wang, W., Ma, L. G., Yang, L. B., et al. (2013). A bipartite molecular module controls cell death activation in the Basal cell lineage of plant embryos. PLoS Biol. 11:e1001655. doi: 10.1371/journal.pbio.1001655

Zhao, P., Zhou, X. M., Zou, J., Wang, W., Wang, L., Peng, X. B., et al. (2014). Comprehensive analysis of cystatin family genes suggests their putative functions in sexual reproduction, embryogenesis, and seed formation. J. Exp. Bot. 65, 5093-5107. doi: 10.1093/jxb/eru274

Zhao, Y., Hu, Y., Dai, M., Huang, L., and Zhou, D. X. (2009). The WUSCHELrelated homeobox gene WOX11 is required to activate shoot-borne crown root development in rice. Plant Cell 21, 736-748. doi: 10.1105/tpc.108.061655

Conflict of Interest Statement: The authors declare that the research was conducted in the absence of any commercial or financial relationships that could be construed as a potential conflict of interest.

The reviewer XZ and handling Editor declared their shared affiliation.

Copyright (c) 2018 Zhou, Guo, Zhao and Sun. This is an open-access article distributed under the terms of the Creative Commons Attribution License (CC $B Y)$. The use, distribution or reproduction in other forums is permitted, provided the original author(s) and the copyright owner are credited and that the original publication in this journal is cited, in accordance with accepted academic practice. No use, distribution or reproduction is permitted which does not comply with these terms. 